\title{
Melorheostosis: A Rare Cause of Scoliosis?
}

\section{Bekir Yavuz Uçar' ${ }^{1 *}$ Meriç Enercan², Mehmet Fethi Ceylan³, Sinan Kahraman², Ahmet Alanay² and Azmi Hamzaoğlu²}

${ }^{1}$ Orthopaedics and Traumatology Department, Dicle University Medical School, Diyarbakır 21280, Turkey

${ }^{2}$ Istanbul Spine Center, Florence Nightingale Hospital, Istanbul 34360, Turkey

${ }^{3}$ Orthopaedics Traumatology Department, Yuzuncu Yil University Medical School, Van, Turkey

\begin{abstract}
Background: To report a case with melorheostosis in the spine associated with scoliosis deformity. To the best of our knowledge, there was no reported case about melorheostosis causing spinal deformities in recent literature.

Methods: This report reviewed the patient`s medical record, her imaging studies and related literature.

Results: A 13-year-old male patient was taken to an orthopaedic clinic by his family three years ago due to low back pain. Patient diagnosed with scoliosis as a result of direct examination and radiographs. When the patient applied to our clinic he was taking analgesic for his pain. The patient was reported frequent episodes of back pain in recent times. He became unable to continue to play his routinely sportive activities. His family was very disturbed and anxious. Spine radiographs showed a slight scoliosis from the T4 level to the T10 level. CT scan demonstrated abnormalities of both bone density and contour in the lumbar spine. Lesions of melorheostosis were consistent with the hyperostosis seen on the CT images. We found melorheostosis causing scoliosis in the patient. We offered detailed information about melorheostosis to the patient and family. Analgesics were regulated and follow-up plans were drawn. The family was very relieved.

Conclusion: Melorheostosis is a rare mesodermal disease and one of the developmental diseases of bone density. The majority of cases describe skeletal abnormalities confined to a single limb, most predominantly the lower extremity. Involvement of and limitation of melorheostosis to the spine is rare. There was no reported case about melorheostosis causing scoliosis in recent literature. Spine surgeons should be alert in terms of pathologies, such as melorheostosis which may cause painful deformities of the spine.
\end{abstract}

Keywords: Scoliosis; Melorheostosis; Spine deformity; Low back pain

\section{Background}

Melorheostosis, also known as Leri's disease which was first described by Leri and Joany in 1922 [1], is a rare, non-inheritable progressive bone dysplasia characterized by irregular cortical hyperostosis. Generally manifests during early childhood, occasionally the initial signs appear in adulthood [2]. It is a benign dysplasia with a very unusual and characteristic roentgenographic appearance. The common reference to this characteristic abnormality is having a "flowing candle wax" appearance [2-7]. This appearance is the result of longitudinal bars of hyperostosis along single or contiguous bones. Ossification may occur in skin and soft tissues overlying the affected bones. The genetic defect in the majority of sporadic and isolated melorheostosis remains unknown. Classically, melorheostosis affects the long bones, especially those in the lower extremities. Involvement of the spine is rare [8-10].

The aim of this case presentation is to report a 13-year-old male patient with melorheostosis in the spine associated with scoliosis deformity. To the best of our knowledge, there was no reported case about melorheostosis causing spinal deformities in recent literature.

\section{Case Presentation}

A 13-year-old male patient was taken to an orthopaedic clinic by his family three years ago due to back pain. Patient diagnosed with scoliosis as a result of direct examination and radiographs were proposed one year follow-up to them and were applied no medical treatment. The patient reported feeling uncomfortable back pain episodes from time to time. The patient had applied in three years to three separate orthopaedic clinics. Follow-up and sporting activities were proposed to the patient. When the patient applied to our clinic he was taking analgesic for his back pain. After obtaining a detailed history, vertebral column orthoroentgenogram was taken. Spine radiographs showed a slight scoliosis (Cobb angle: $20^{\circ}$ ) from the T4 level to the T10 level (Figure 1).

The patient reported frequent episodes of back pain in recent times. He became unable to play his routinely sportive activities. His family was very disturbed and anxious. Detailed investigations were done. The patient underwent a magnetic resonance imaging (MRI) and then was referred to take a computed tomography scan (CT) of his vertebral column. CT scan demonstrated abnormalities of both bone density and contour in the lumbar spine. Lesions of melorheostosis were consistent with the hyperostosis seen on the CT images (Figure 2).

We found melorheostosis causing scoliosis in the patient. There was not an indication that required surgical treatment. We offered detailed information about melorheostosis to the patient and family. Analgesics were regulated and follow-up plans were drawn. The family was very relieved.

\section{Conclusions}

Melorheostosis is a rare mesodermal disease and one of the developmental diseases of bone density. This disorder tends to be segmental and unilateral and may affect only one bone (monostotic), one limb (monomelic), or multiple bones (polyostotic) [1,4,6]. Until

*Corresponding author: Bekir Yavuz Uçar, Orthopaedics and Traumatology Department, Dicle University Medical School, Diyarbakır 21280, Turkey, E-mail: drbyucar@yahoo.com

Received October 29, 2012; Accepted December 20, 2012; Published December 22, 2012

Citation: Uçar BY, Enercan M, Ceylan MF, Kahraman S, Alanay A, et al. (2013) Melorheostosis: A Rare Cause of Scoliosis? J Spine 2: 133. doi:10.4172/21657939.1000133

Copyright: (C) 2013 Uçar BY, et al. This is an open-access article distributed under the terms of the Creative Commons Attribution License, which permits unrestricted use, distribution, and reproduction in any medium, provided the original author and source are credited. 


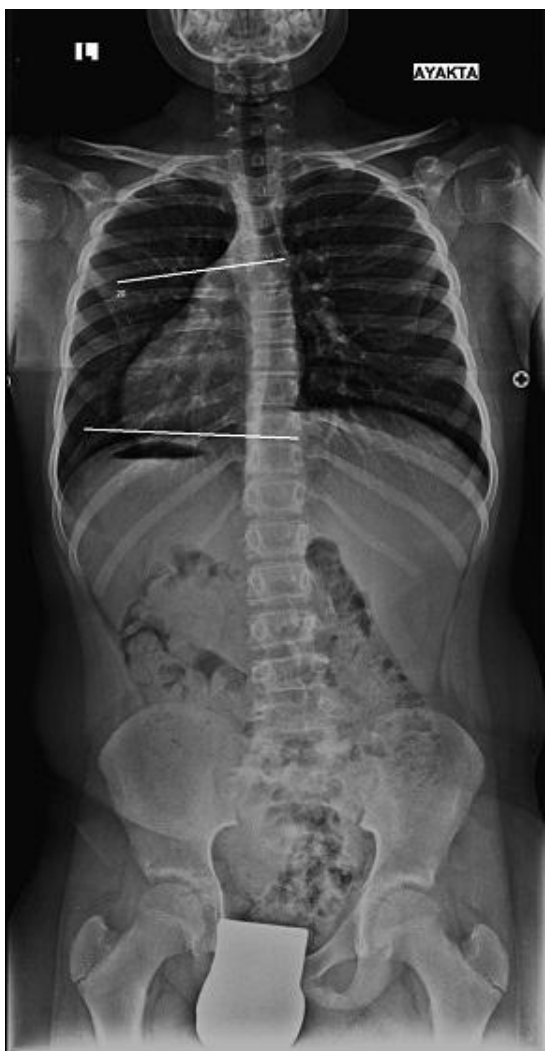

Figure 1: PA radiographs showed a slight scoliosis (Cobb angle: $20^{\circ}$ ) from the T4 level to the T10 level.

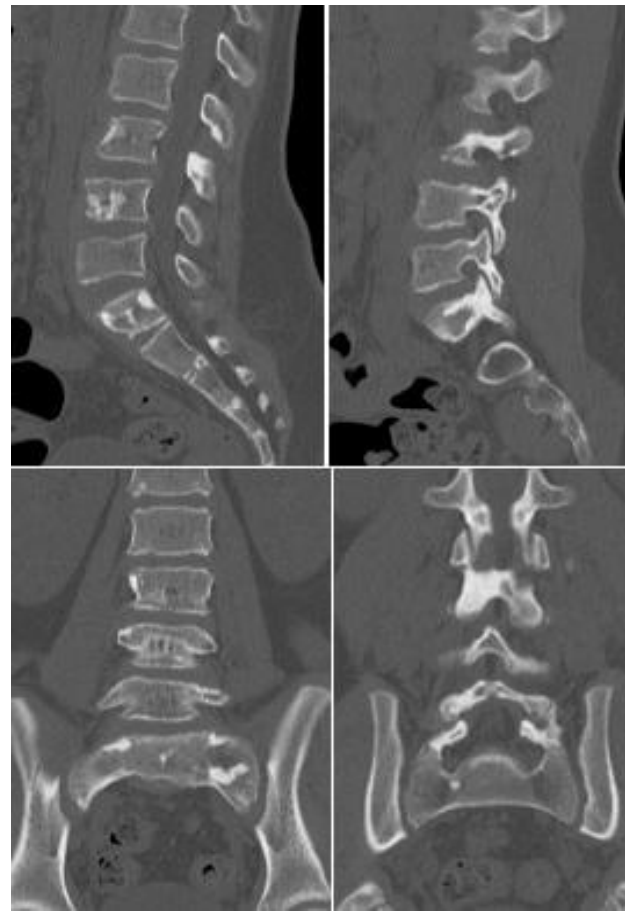

Figure 2: CT scan demonstrated abnormalities of bone density and contour in the lumbar spine. Lesions of melorheostosis were consistent with the hyperostosis seen on the CT images (sagittal and coronal). very recently, the etiology of melorheostosis was unknown. It has been established that melorheostosis is due to a loss of function mutation in LEMD3 gene (known as MAN1), which encodes an inner nuclear membrane protein. Age at presentation varies widely and melorheostosis may be seen in children and adults. Its incidence is estimated at 0.9 per million, with no sex preference [2]. The disease is insidious, with a slow chronic progression of symptoms and periodic exacerbations. The usual clinical presentation consists of chronic bone pain and joint contractures [7]. The majority of cases describe skeletal abnormalities confined to a single limb, most predominantly the lower extremity. Melorheostosis rarely involves the spine, skull, and facial bones $[1,4,6]$. When the disease involves the appendicular skeleton, it may be asymptomatic or give symptoms of pain and limitation of joint movement $[2,8-10]$.

The diagnosis of melorheostosis was made on the basis of the characteristic distribution, location, and combined radiographic, CT, and MR imaging features of the abnormalities [11]. Plain radiographs are sufficient to make the diagnosis, although computed tomography and magnetic resonance imaging are very helpful in the analysis of the cortical thickening and its extension to the medullary canal of bones. The radiographic view, the classic pathognomonic pattern is the so-called "dripping candle wax", figurative description for longitudinal bars of hyperostosis along the cortical bone of long bones. CT can confirm the presence of calcifications inside the masses and demonstrate their topography. It is often found that they are not directly attached to the bones.

Involvement of and limitation of melorheostosis to the spine is rare. Most of the reported cases of spinal melorheostosis were asymptomatic [4]. Spinal melorheostosis may present with significant stenosis of the vertebral canal caused either by the hyperostosis or due to associated fibrolipomatous lesions resulting neurological impairment [12]. Zeiller et al. reported a case with severe spinal stenosis of the lower cervical and upper thoracic spine due to melorheostosis, resulting in progressive myelopathy which required a surgical decompression [13]. Yoon et al. reported another case with melorheostosis of the sacrum causing acute-onset neurological symptoms [14].

Spinal melorheostosis may also be present with significant back pain causing disability. Robertson et al. reported spinal melorheostosis affecting lumbar vertebrae with involvement of facet joints causing severe back pain which responded favourably to decompression and fusion surgery [5]. McCarthy et al. also reported a case of melorheostosis involving the tenth and eleventh thoracic vertebrae crossing the facet joint presenting with back pain [15].

There was no reported case about melorheostosis causing scoliosis in recent literature. Spine surgeons should be alert in terms of pathologies, such as melorheostosis which may cause painful deformities of the spine.

Treatment of spinal melorheostosis is nonoperative in most cases $[3,6]$. Surgery may need because of abnormal bony architecture [5].

\section{Consent}

Written informed consent was obtained from the patient for publication of this case report and any accompanying images.

\section{Competing Interests}

The authors of this paper do not have any conflict of interest, financial or nonfinancial, related with the study.

\section{Acknowledgements}

N/A 
Citation: Uçar BY, Enercan M, Ceylan MF, Kahraman S, Alanay A, et al. (2013) Melorheostosis: A Rare Cause of Scoliosis? J Spine 2: 133. doi:10.4172/2165-7939.1000133

Page 3 of 3

\section{Authors' Contribution}

All authors participated in the design and redaction of the text and conclusions. All authors read and approved the final manuscript.

\section{References}

1. Leri A, Joanny $J$ (1992) Une affection non de'crite des os hyperostose "en coule'e" sur toute la longeur d'un member ou "melorhe'ostose". Bull Memoires Soc Med Hopitaux Paris 46: 1141-1145

2. Campbell CJ, Papademetriou T, Bonfiglio M (1968) Melorheostosis. A report of the clinical, roentgenographic, and pathological findings in fourteen cases. J Bone Joint Surg Am 50: 1281-1304.

3. Younge D, Drummond D, Herring J, Cruess RL (1979) Melorheostosis in children. Clinical features and natural history. J Bone Joint Surg Br 61B: 415418.

4. Kalbermatten NT, Vock P, Rüfenacht D, Anderson SE (2001) Progressive melorheostosis in the peripheral and axial skeleton with associated vascular malformations: imaging findings over three decades. Skeletal Radiol 30: 48-52.

5. Robertson PA, Don AS, Miller MV (2003) Painful lumbosacral melorheostosis treated by fusion. Spine 28: E234-238

6. Khurana JS, Ehara S, Rosenberg AE, Rosenthal DI (1988) Case report 510: Melorheostosis of ilium, femur, and adjacent soft tissues. Skeletal Radiol 17: $539-541$
7. Jain VK, Arya RK, Bharadwaj M, Kumar S (2009) Melorheostosis clinicopathological features, diagnosis, and management. Orthopedics 32: 512.

8. Beauvais P, Fauré C, Montagne JP, Chigot PL, Maroteaux P (1977) Leri's melorheostosis: three pediatric cases and a review of the literature. Pediat Radiol 6: 153-159.

9. Hove E, Sury B (1971) Melorheostosis. Report on 5 cases with follow-up. Acta Orthop Scand 42: 315-319.

10. Werner MS, Scheimer RA (1987) Melorheostosis. A review of the literature and case report. J Am Podiatr Med Assoc 77: 96-98.

11. Motimaya AM, Meyers SP (2006) Melorheostosis involving the cervical and upper thoracic spine: radiographic, $\mathrm{CT}$, and MR imaging findings. AJNR Am J Neuroradiol 27: 1198-1200

12. Suresh S, Muthukumar T, Saifuddin A (2010) Classical and unusual imaging appearances of melorheostosis. Clin Radiol 65: 593-600.

13. Zeiller SC, Vaccaro AR, Wimberley DW, Albert TJ, Harrop JS, et al. (2005) Severe myelopathy resulting from melorheostosis of the cervicothoracic spine. A case report. J Bone Joint Surg Am 87: 2759-2762.

14. Yoon J, Al Shafai L, Nahal A, Turcotte RE, Martin MH (2011) Melorheostosis of the sacrum causing acute-onset neurological symptoms. Skeletal Radiol 40 : 1369-1373.

15. McCarthy M, Mehdian H, Fairbairn KJ, Stevens A (2004) Melorheostosis of the tenth and eleventh thoracic vertebrae crossing the facet joint: a rare cause of back pain. Skeletal Radiol 33: 283-286. 